Running head: DEMQOL-Proxy (self-administered)

\title{
Reliability and validity of a self-administration version of DEMQOL-Proxy
}

\author{
A.A. Jolijn Hendriks ${ }^{1}$, Sarah C. Smith ${ }^{1}$, Theopisti Chrysanthaki ${ }^{1}$ and Nick Black ${ }^{1}$ \\ ${ }^{1}$ London School of Hygiene and Tropical Medicine
}

\section{Key Words}

HRQL; dementia; DEMQOL-Proxy; self-administration; psychometric properties; validity; reliability

\section{Key Points}

- DEMQOL-Proxy (self-administered) has comparable acceptability, reliability and validity with DEMQOL-Proxy (interviewer-administered)

- It is feasible to use DEMQOL-Proxy (self-administered) in a wider variety of contexts, including routine use in busy clinics

- DEMQOL-Proxy (self-administered) should still be used alongside DEMQOL

\section{Funding}

This research was commissioned and funded by the Department of Health Policy Research Programme (Using Patient Reported Outcome Measures to Assess Quality of Life in Dementia, 0700071). The views expressed in this publication are those of the authors and not necessarily those of the Department of Health.

\section{No conflicts of interest}

Word count body of text: 3106

\section{Corresponding author:}

A.A.J. Hendriks, Department of Health Services Research and Policy, London School of Hygiene and Tropical Medicine, 15-17 Tavistock Place, London WC1H 9SH, United Kingdom. Phone: +44 (0)20 7927 2724. Fax: +44 (0)20 7927 2701. Email address: jolijn.hendriks@1shtm.ac.uk. 


\begin{abstract}
Objective: This study aimed to investigate the reliability and validity of a self-administered version of DEMQOL-Proxy, a disease specific instrument that measures health-related quality of life (HRQL) in people with dementia.

Methods: The sample consisted of 173 informal carers of people with dementia, aged 29 to 89 years old. Carers were mostly female, White/White British and closely related to the patient. They completed DEMQOL-Proxy (self-administered), the EQ-5D-3L (proxyreported about the person with dementia), EQ-5D-3L (self-reported about their own health) and the Zarit Carer Burden Index (ZBI). Using well-established methods from Classical Test Theory we evaluated scale level acceptability, reliability and convergent, discriminant and known-groups validity of DEMQOL-Proxy.
\end{abstract}

Results: DEMQOL-Proxy (self-administered) showed high acceptability (3.5\% missing data and $0 \%$ scores at floor or ceiling), high internal consistency reliability $(\alpha=0.93)$ and good convergent and discriminant validity. Amongst others, we found a moderately high correlation with EQ-5D-3L Proxy-reported $(r=0.52)$ and low to essentially zero correlations with EQ-5D-3L Self-reported $(r=0.20)$ and carer and patient background variables $(r \leq$ 0.20). As predicted, DEMQOL-Proxy (self-administered) showed a modest correlation with DEMQOL $(r=0.32)$. Known-groups differences on HRQL (comparing people with versus people without cognitive impairment) were of moderate effect size $(d=0.38)$ and in the expected direction.

Conclusions: DEMQOL-Proxy (self-administered) has comparable acceptability, reliability and validity with DEMQOL-Proxy (interviewer-administered). DEMQOL-Proxy (selfadministered) can be used in a wider variety of contexts than its interviewer-administered version, including routine use in busy clinics. 


\section{Introduction}

Patient-reported outcome measures (PROMs) are well established in clinical trials and health services research as important ways of measuring patients' perceptions and understanding of their health condition and treatment (e.g. Browne et al., 2008; Dawson et al., 2010). The Department of Health (England) is also committed to ensuring that assessing the outcome of care takes into account the views of patients and, where relevant, their lay carers (Black, 2013). PROMs are routinely collected for four areas of elective operations (hip and knee replacement, varicose surgery and hernia repair) in England and development studies have been or are being conducted for a number of more long term conditions, including dementia (National PROMs Programme - http://www.hscic.gov.uk/).

DEMQOL and DEMQOL-Proxy (Smith et al., 2005a, 2005b, 2007) are disease specific instruments that measure important aspects of health-related quality of life (HRQL) in people with dementia. DEMQOL is self-reported by the person with dementia and DEMQOL-Proxy is reported by their family (informal) carer on behalf of the person with dementia. Both instruments are interviewer administered according to a standard manual and were developed to be used together to provide complementary perspectives; DEMQOLProxy was not intended as a substitute for the reports of the person with dementia but is a practical alternative in later stages of the condition when self-report may no longer be possible. There is reported evidence of reliability and validity for both instruments in people with mild or moderate dementia. Furthermore, although the evidence is based on a small sample, DEMQOL-Proxy is also promising with people with severe dementia (Smith et al., 2005a, 2007). Recently, Mulhern et al. (2013) developed disease-specific utility scores for DEMQOL and DEMQOL-Proxy. The two instruments are widely used in research and are currently being considered for routine use by the Department of Health (England) (Department of Health, 2014, p. 37). 
The practicality of using DEMQOL-Proxy in routine contexts may be limited by the need for interviewer administration. This form of administration was originally deemed necessary as many family carers were themselves elderly, but it is time consuming and labour intensive. In this paper, we present the psychometric evaluation of DEMQOL-Proxy (selfadministered), an instrument that is still intended to be used alongside DEMQOL, but the simpler administration method is likely to be more feasible for use in routine settings. Although so-called "modern" psychometric methods are increasingly used in the development of health outcome measures and have many advantages, we used classical psychometric methods to investigate acceptability, reliability and validity of DEMQOLProxy (self-administered). This was because we sought to compare the self-administered version of DEMQOL-Proxy with the original interviewer administered version using the same methods of analysis.

\section{Methods}

\section{Participants}

As part of the pilot phase of a large study evaluating Memory Assessment Services (MAS) we recruited 173 informal carers of people with dementia who were attending one of four MAS (Barnet, Enfield and Haringey; West London; Humber; and South West Yorkshire) for a first referral for suspected dementia and who had agreed to be part of the study. Patients who had insufficient English language to be able to understand the consent process or complete the questionnaires were excluded (as were their carers). Sample characteristics are presented in Table 1. Carers were mostly female, White/White British and were the patient's partner (husband/wife/partner) or their son or daughter. Carers ranged in age from 29 to 89 years $($ mean $=62, S D=14)$. We categorised disease severity based on patients' scores on the Mini-Mental State Examination (MMSE; Folstein et al., 1975), using a cut point of < 24 (or 
an equivalent published cut-off score if a different measure was used) to indicate the presence of cognitive impairment (Tombaugh and McIntyre, 1992). Based on this categorisation, 56\% of the carers cared for a patient with cognitive impairment and 33\% cared for a patient with no cognitive impairment; for $11 \%$ of the carers cognitive impairment level of the patient they cared for is unknown.

[Insert Table 1 about here]

\section{Procedure}

Patients and their carers were sent an invitation letter introducing the study with their clinic appointment letter. Written, informed consent was obtained from both the patient and their carer at their clinic appointment (held either in the clinic or at home). The carer was asked to complete DEMQOL-Proxy in self-administered format (i.e. carers were given the questionnaire booklet and asked to complete it by themselves). All instructions to the carer were standardised, i.e. in written format in the questionnaire only. No additional instructions were provided except for asking the carer to read the written instructions and complete the questionnaire without consulting the patient. In addition to DEMQOL-Proxy, carers also completed the EQ-5D (proxy-reported about the person with dementia and also self-reported about their own health) and the Zarit Carer Burden Index (see Instruments below). Afterwards patients were asked to complete DEMQOL, using the usual interviewer administered format. Ethical approval was obtained from the National Research Ethics Service Committee London - Queen Square (REC Reference Number: 14/LO/1146).

\section{Instruments}

DEMQOL-Proxy. DEMQOL-Proxy (Smith et al., 2005a, 2005b, 2007) consists of 31 questions each assessed on a 4-point response scale: a lot, quite a bit, a little, not at all. The questions were derived from five conceptual domains: health and well-being (12 
questions), cognitive functioning (9 questions), daily activities (6 questions), social relationships (2 questions) and self-concept ( 2 questions). There is also an additional overall quality of life question, answered on a 4-point scale: very good, good, fair, poor. The items are scored according to a standard scoring algorithm (http://www.bsms.ac.uk/research/ourresearchers/sube-banerjee/demqol/) to produce an overall score where higher scores represent better HRQL.

EQ-5D-3L. The EQ-5D (Brooks, 1996; The EuroQol Group, 1990) is a generic measure of health utility, that is widely used to evaluate patient-reported health outcome in routine contexts. It was therefore included in this study as a validating measure for DEMQOL-Proxy and so as to be able to compare the results with similar studies. The EQ5D includes one question on each of 5 domains: mobility, self-care, usual activities, pain/discomfort and anxiety/depression and also a visual analogue scale represented by a thermometer running from 0 (worst imaginable health state) to 100 (best imaginable health state). Each of the 5 descriptive questions has its own 3-point response scale. Carers completed the EQ-5D about their own health and also as a proxy-report about the person with dementia (where they were asked to give the answer they thought the patient would give). We used the UK TTO based index values (Dolan, 1997; MVH Group, 1995).

Zarit Burden Interview (ZBI). The short version of the Zarit Burden Interview consists of 12 items that assess carer burden (Bédard et al., 2001). All of the questions are reported on a 5-point Likert-type response scale: 0 (never), 1 (rarely), 2 (sometimes), 3 (quite frequently) or 4 (nearly always). Item scores are summed to create an overall score where higher scores represent more carer burden. Additionally, two subscale scores can be created: Personal Strain (items 1 to 9) and Role Strain (item 10 to 12). Because we made one minor change to the wording of the questionnaire (changing the word "relative" to "relative/friend" to allow for the possibility that carers may not be family members), we checked whether we 
replicated this two-dimensional structure in our data set; details can be obtained from the first author. Internal consistency reliability Cronbach's $\alpha$ was high (Total score: 0.93, Personal Strain: 0.94, Role Strain: 0.83). We concluded our re-worded version of the short version of the ZBI to be appropriate for use in this study.

\section{Psychometric analyses}

The psychometric analyses aimed to replicate the psychometric evaluation undertaken on the original interviewer-administered version of DEMQOL-Proxy (Smith et al., 2005a). This was based on well-established methods from Classical Test Theory (CTT) (Nunnally and Bernstein, 1994; Scientific Advisory Committee of the Medical Outcomes Trust, 2002; US Food and Drug Administration, 2009) and evaluated its scale level acceptability, reliability and validity. Where possible we used the same validity hypotheses as were used to evaluate the original interviewer-administered form of DEMQOL-Proxy, though in some instances these have been adapted as the present study did not include other dementia-specific HRQL instruments and also used a different generic measure (EQ-5D instead of the SF-12).

Acceptability and Data Quality. Acceptability refers to completeness of the data, i.e. the proportion of the sample for whom an overall score on DEMQOL-Proxy can be computed. To obtain scores we applied the instrument's standardised scoring algorithm, which means that for cases with less than $50 \%$ missing item scores, missing values were replaced by the person specific mean across completed items. Data quality refers to the overall score having sufficient variance and no large floor and ceiling effects (large proportions of the sample with lowest/highest overall score preventing measuring deterioration/improvement). The criterion for acceptability was $<5 \%$ missing data for the overall score, the criterion for data quality was floor and ceiling effects $\leq 15 \%$ (cf. Lim et al., 2015). 
Reliability (Internal Consistency). Internal consistency reliability indicates the extent to which the items have something in common and each item contributes positively to the overall scale. It was evaluated using Cronbach's alpha (Cronbach, 1951) (criterion: $\alpha \geq$ $0.7)$.

Convergent Validity. Convergent validity is the extent to which a construct is correlated with measures of the same or similar constructs. We examined convergent validity of DEMQOL-Proxy (self-administered) in three ways: Firstly, we examined the correlation between DEMQOL-Proxy (self-administered) and DEMQOL. Like DEMQOL, DEMQOLProxy purports to assess the patient's HRQL, however the strength of the association between these two scales is known to be modest (Smith et al., 2005b). Based on findings with the interviewer administered version of DEMQOL-Proxy (Smith et al., 2005a), we expected a correlation of 0.3 to 0.4 . Secondly, we examined the relationship between DEMQOL-Proxy (self-administered) and the EQ-5D. We expected a moderate positive correlation in the region of 0.3-0.5 between these two instruments (as one is disease specific and the other is generic so the strength of relationship may be reduced). Thirdly, we examined the relationship between DEMQOL-Proxy (self-administered) and the ZBI (short version). We hypothesised that higher carer burden would be associated with lower HRQL of the person with dementia, however only moderately (in the region of 0.3-0.5), as these are similar but different constructs.

Discriminant Validity. Discriminant validity is the extent to which a construct is uncorrelated with measures of different constructs. We examined discriminant validity by comparing DEMQOL-Proxy (self-administered) with the EQ-5D ratings that the carers made about themselves. We hypothesised that DEMQOL-Proxy should not be correlated with the carer's own health. We also examined the association between DEMQOL-Proxy and both carer's background variables (age, gender, number of years acquainted, hours spent with the 
patient in the last week) and patient characteristics (age and gender); we hypothesised no associations.

Known Groups Validity. Known-groups validity is the extent to which the construct shows expected differences between groups that are known to be different. Little is known about the natural history of HRQL in dementia and how it is expected to change with increasing severity, though there is some evidence, based on small samples for differences between mild/moderate and severe (Smith et al. 2005a). As very few of our sample had severe cognitive impairment, we investigated the hypothesis that people without cognitive impairment (MMSE score $\geq 24$ ) would have higher DEMQOL-Proxy scores than people with cognitive impairment (MMSE score < 24). Effect size was assessed by Cohen's d (Cohen, 2009).

\section{Results}

\section{Acceptability and Data Quality}

Table 2 provides information on acceptability and data quality. Acceptability was high for DEMQOL-Proxy (self-administered). There were only a small number of missing scores (3.5\%), though in part this reflects the imputation rule that is part of the scoring algorithm for DEMQOL-Proxy, and there were no floor or ceiling effects. DEMQOL-Proxy scores ranged from 38 to $123($ mean $=86.3, S D=16.1)$. The distribution of DEMQOL-Proxy total score appeared slightly skewed to the left (-0.47), with the mass of the distribution concentrated above the mean $($ median $=89)$.

[Insert Table 2 about here]

\section{Reliability (Internal Consistency)}


DEMQOL-Proxy (self-administered) had high internal consistency (Cronbach's $\alpha=0.93, N$ $=167)$, slightly higher than in the original development for the interviewer-administered version of DEMQOL-Proxy ( $\alpha=0.89$; Smith et al., 2005a, p. 54).

\section{Convergent validity}

The association between DEMQOL-Proxy (self-administered) and DEMQOL was 0.32, in agreement with expectations. Furthermore, as hypothesised, DEMQOL-Proxy (selfadministered) showed a moderately high positive association with carer's proxy rating of HRQL on the EQ-5D $(r=0.52)$. In addition, DEMQOL-Proxy (self-administered) showed a weaker negative correlation with ZBI ( $r=-0.41$ to $r=-0.46$ ), although the association was slightly stronger than expected. See Table 3 for the convergent (and discriminant) correlations.

[Insert Table 3 about here]

\section{Discriminant validity}

We found a weak, positive association between DEMQOL-Proxy (self-administered) and carer's self-rating on the EQ-5D $(r=0.20)$, see Table 3. The difference with the convergent correlation of DEMQOL-Proxy (self-administered) with carer's proxy-rating on the EQ-5D ( $r$ $=0.52$ ) is statistically significant, $z=3.58, p<0.001$ (Lee and Preacher, 2013; Steiger, 1980). Furthermore, we found a weak correlation with carer's age $(r=0.20)$; all other associations of DEMQOL-Proxy (self-administered) with background variables of carer and patient were essentially zero (Table 3). We found no statistically significant differences in DEMQOLProxy (self-administered) total score for male and female carers, mean $=85.8(\mathrm{SD}=15.7), n$ $=51$ and mean $=86.4(\mathrm{SD}=16.0), n=113$, respectively, $t(162)=-0.21, p=0.82$ (two-tailed), effect size $=0.04$. Likewise, we found no statistically significant differences in DEMQOL-Proxy (self-administered) total score for male and female patients, mean $=87.6$ 
$(\mathrm{SD}=16.5), n=76$ and mean $=85.0(\mathrm{SD}=15.3), n=88$, respectively, $t(162)=1.06, p=$ 0.29 (two-tailed), effect size $=0.16$.

\section{Known Groups Differences}

DEMQOL-Proxy (self-administered) showed known-groups validity as, on average, people without cognitive impairment (mean $=89.9, S D=14.6, n=57$ ) had better HRQL scores than people with cognitive impairment $($ mean $=84.0, S D=16.6, n=92), t(147)=2.20, p=0.015$ (one-tailed), effect size $=0.38$ (Table 2$)$.

\section{Discussion}

The present study aimed to investigate the reliability and validity of a self-administered version of DEMQOL-Proxy compared with the original interviewer administered version (Smith et al., 2005a, 2007). As with the original interviewer administered version we used CTT to investigate DEMQOL-Proxy (self-administered)'s psychometric properties. Overall, the results suggest that the two versions are comparable.

As for the original version, acceptability of DEMQOL-Proxy (self-administered) was high (little missing data and no floor/ceiling effects). Reliability was assessed in terms of internal consistency. The high Cronbach's alpha indicated that all of the items were homogenous and relating to a similar underlying construct. Convergent validity (moderate association with DEMQOL, EQ-5D and three ZBI scores) and discriminant validity hypotheses (little or no association between DEMQOL-Proxy and carer's own health, carer's age, gender, person with dementia's age, gender, length of time that the carer had known the person with dementia or the amount of time spent with them each week) were supported. Known-groups validity was supported by a moderate effect size between groups with and without cognitive impairment. 
The results support the use of DEMQOL-Proxy as a self-administered questionnaire. Moreover, the analyses reported here support a more practical use of DEMQOL-Proxy and enable it to be used in a more flexible way. Self-report is potentially less time consuming and cheaper than interviewer administration. Given the growing interest in the use of patient reported outcome measures (PROMS) as part of routine monitoring of health care including dementia care (Department of Health, 2009), this is an important, practical development. The DEMQOL system is one of the few disease-specific HRQL measures for people with dementia that consists of both patient and proxy report. It can therefore be used across the range of severity and types of dementia and across different care arrangements. Where possible both patient and proxy reports should be collected; it keeps the patient's view central while complementing it with the proxy's view. The development of DEMQOL-Proxy (selfadministered) makes the use of DEMQOL-Proxy more feasible, particularly in in busy clinics.

The analyses reported here have some limitations. We have not evaluated test-retest reliability as collection of these was not feasible within the design of this study. This should be considered before the psychometric robustness of DEMQOL-Proxy (self-administered) can be confirmed. The clinic setting (as opposed to sending questionnaires by mail) meant that there was a member of staff available to respond to questions if necessary. None of the four sites reported that carers had difficulty completing the questionnaires, and clinic staff were instructed not to interpret or re-phrase questions. However, it is possible that different responses to queries in different clinics could affect the reliability of DEMQOL-Proxy (selfadministered). The results are also limited by the high ceiling effects found for the EQ-5D Self-rating. We used the EQ-5D as a validating measure as it is widely used in other studies that have evaluated the feasibility of PROMs in routine settings. However, the large proportion of the sample who scored the maximum score restricted the range and reduced 
discrimination for EQ-5D Self-rating more than for EQ-5D Proxy-rating, which may have artificially attenuated the discriminant correlation.

Finally, these analyses are also based on psychometric methods derived from CTT. Although these methods are well established (Scientific Advisory Committee of the Medical Outcomes Trust, 2002; US Food and Drug Administration. 2009), their limitations for health related scores have become well known over the last 10 years (Hobart and Cano, 2009). In particular, the scores derived from these methods are ordinal rather than interval, they are specific to the particular sample in which they are derived and they can only be used at the group level. We used these methods so as to first be able to compare directly with the analyses from the development of the original interviewer administered version of DEMQOL-Proxy, using the same methods. Our current work will use modern psychometrics (Rasch Measurement Theory; Rasch, 1960/1980) to re-evaluate the measurement properties of both DEMQOL and DEMQOL-Proxy (self-administered) and to improve the scoring algorithms.

\section{Conclusions}

DEMQOL-Proxy (self-administered) has comparable acceptability, reliability and validity with DEMQOL-Proxy (interviewer-administered). It is consequently feasible to use DEMQOL-Proxy (self-administered) in a wider variety of contexts, including routine use in busy clinics. Robust use of the instrument will still depend on careful training of staff to ensure that its use is standardised. This includes training staff not to interpret or re-phrase questions, even if they are asked. DEMQOL-Proxy (self-administered) should be scored using the standard scoring algorithm to derive a single overall score (http://www.bsms.ac.uk/research/our-researchers/sube-banerjee/demqol/). Where possible DEMQOL-Proxy (self-administered) should still be used alongside DEMQOL and the two instruments should be interpreted together. 


\section{References}

Bédard M, Molloy DW, Squire L, Dubois S, Lever JA, O'Donnell M. 2001. The Zarit Burden Interview: A new short version and screening version. Gerontologist 41: 652-657. Black N. 2013. Patient reported outcome measures could help transform healthcare. $B M J$ 346: $19-21$.

Brooks R. 1996. EuroQol: the current state of play. Health Policy 37: 53-72.

Browne J, Jamieson L, Lewsey J, Van der Meulen J, Copley L, Black N. 2008. Case-mix \& patients' reports of outcome in Independent Sector Treatment Centres: comparison with NHS providers. BMC Health Services Res 8: 78.

Cohen J. 2009. Statistical power analysis for the behavioural sciences. $2^{\text {nd }}$ ed. Psychology Press: New York; pp. 24-27, 79-81.

Cronbach LJ. 1951. Coefficient alpha and the internal structure of tests. Psychometrika 16: 297-334.

Dawson J, Doll H, Fitzpatrick R, Jenkinson C, Carr AJ. 2010. The routine use of patient reported outcome measures in healthcare settings. BMJ 340: c186.

Department of Health. 2009. Living well with dementia: A National Dementia Strategy. Department of Health: London.

Department of Health. 2014. The Adult Social Care Outcomes Framework 2015/16. Department of Health: London. https://www.gov.uk/government/uploads/system/uploads/attachment_data/file/375431 /ASCOF_15-16.pdf

Dolan P. 1997. Modeling valuations for EuroQol health states. Med Care 35: 1095-1108. Folstein MF, Folstein SE, McHugh PR. 1975. "Mini-mental state". A practical method for grading the cognitive state of patients for the clinician. J Psychiatr Res 12: 189-198. 
Hobart J, Cano S. 2009. Improving the evaluation of therapeutic interventions in multiple sclerosis: the role of new psychometric methods. Health Technol Assess 13(12).

Lee IA, Preacher KJ. 2013, September. Calculation for the test of the difference between two dependent correlations with one variable in common [Computer software]. Available from http://quantpsy.org.

Lim CR, Harris, K, Dawson J, et al. (2015). Floor and ceiling effects in the OHS: an analysis of the NHS PROMs data set. BMJ Open 5:e007765. DOI: 10.1136/bmjopen-2015007765.

Mulhern B, Rowen D, Brazier J, et al. 2013. Development of DEMQOL-U and DEMQOLPROXY-U: generation of preference-based indices from DEMQOL and DEMQOLPROXY for use in economic evaluation. Health Technol Assess 17(5).

MVH Group. 1995. The Measurement and Valuation of Health. Final Report on the Modelling of Valuation Tariffs. MVH Group, Centre for Health Economics: York.

Nunnally JC, Bernstein IH. 1994. Psychometric Theory. $3^{\text {rd }}$ ed. McGraw-Hill: New York.

Rasch G. 1960/1980. Probabilistic Models for Some Intelligence and Attainment Tests. Danish Institute for Education Research: Copenhagen; Expanded edition 1980. University of Chicago Press: Chicago; (reprinted 1993, available from www.rasch.org/books.htm).

Scientific Advisory Committee of the Medical Outcomes Trust. 2002. Assessing health status and quality-of-life instruments: attributes and review criteria. Qual Life Res 11: 193-205.

Smith SC, Lamping DL, Banerjee S, et al. 2005a. Measurement of health-related quality of life for people with dementia: development of a new instrument (DEMQOL) and an evaluation of current methodology. Health Technol Assess 9(10). 
Smith SC, Lamping DL, Banerjee S, et al. 2007. Development of a new measure of health-related quality of life for people with dementia: DEMQOL. Psychol Med 37: $1-10$.

Smith SC, Murray J, Banerjee S, et al. 2005b. What constitutes health-related quality of life in dementia? Development of a conceptual framework for people with dementia and their carers. Int J Ger Psychiatry 20: 889-895.

Steiger JH. 1980. Tests for comparing elements of a correlation matrix. Psychol Bull 87: 245-251.

The EuroQol Group. 1990. EuroQol - a new facility for the measurement for health-related quality of life. Health Policy 16: 199-208.

Tombaugh TN, McIntyre NJ. 1992. The Mini-Mental State Examination: A comprehensive review. J Am Geriatr Soc 40: 922-935.

US Food and Drug Administration. 2009. Guidance for industry on patient-reported outcome measures: Use in medicinal product development to support labeling claims. Federal Register 74: 1-43. 
Table 1

Respondent Characteristics

\begin{tabular}{lrrrrrr}
\hline & & \multicolumn{2}{c}{ Carer } & \multicolumn{2}{c}{ Patient } \\
\hline \multirow{3}{*}{ Gender } & Male & 52 & $(31.3)$ & 78 & $(47.0)$ \\
& Female & 114 & $(68.7)$ & 88 & $(53.0)$ \\
\multirow{2}{*}{ Age } & & & & & \\
& Mean $(S D)$ & 62.0 & $(13.7)$ & 78.7 & $(8.1)$ \\
& Range $(n)$ & $29-89$ & $(164)$ & $52-93$ & $(166)$
\end{tabular}

\section{Ethnicity}

White/White British

Black/Black British

Asian/Asian British

Mixed

Other

$\begin{array}{rrrr}149 & (90.9) & 148 & (90.2) \\ 1 & (0.6) & 1 & (0.6) \\ 10 & (6.1) & 11 & (6.7) \\ 3 & (1.8) & 0 & (0.0) \\ 1 & (0.6) & 4 & (2.4)\end{array}$

\section{Relationship to patient}

Husband/wife/partner

Son/daughter

Son/daughter-in-law

Sibling

Other relative

Friend

Neighbour

Other

$\begin{array}{rr}73 & (44.2) \\ 66 & (40.0) \\ 13 & (7.9) \\ 5 & (3.0) \\ 3 & (1.8) \\ 3 & (1.8) \\ 1 & (0.6) \\ 1 & (0.6)\end{array}$

\section{Living with patient}

$\begin{array}{lll}\text { Yes } & 87 & (52.7) \\ \text { No } & 78 & (47.3)\end{array}$

\section{Years acquainted}

$\begin{array}{rrr}\text { Mean }(S D) & 49.5 & (13.7) \\ \text { Range }(n) & 1-80 & (164)\end{array}$

Days spent (last week)

$\begin{array}{rrr}\text { Mean }(S D) & 5.2 & (2.3) \\ \text { Range }(n) & 0-7 & (165)\end{array}$

Hours spent (last week)

$\begin{array}{rrr}\text { Mean }(S D) & 80.6 & (73.6) \\ \text { Range }(n) & 0-168 & (160)\end{array}$

Note. Unless stated otherwise, the entries are numbers followed by percentages (in parentheses). 
Table 2

Acceptability and Data Quality

\begin{tabular}{|c|c|c|c|c|c|c|c|c|c|}
\hline Scales & $n$ & $\begin{array}{c}\text { Missing } \\
n(\%)\end{array}$ & Scale & Sample & $\%$ Floor & $\%$ Ceiling & Mean & $(S D)$ & Skew \\
\hline \multicolumn{10}{|l|}{ Whole sample ${ }^{a}$} \\
\hline DEMQOL Proxy & 167 & $6(3.5)$ & $31-124$ & $38-123$ & 0.0 & 0.0 & 86.3 & $(16.1)$ & -0.47 \\
\hline EQ-5D Proxy & 162 & $11(6.4)$ & $-0.594-1$ & $-0.429-1$ & 0.0 & 9.3 & 0.579 & $(0.303)$ & -0.94 \\
\hline EQ-5D Self & 163 & $10(5.8)$ & $-0.594-1$ & $-0.594-1$ & 0.6 & 46.0 & 0.806 & $(0.279)$ & -2.29 \\
\hline ZBI Total score & 158 & $15(8.7)$ & $0-48$ & $0-48$ & 6.3 & 0.6 & 13.5 & $(9.9)$ & 0.94 \\
\hline ZBI Personal Strain & 158 & $15(8.7)$ & $0-36$ & $0-36$ & 12.0 & 0.6 & 9.2 & $(8.0)$ & 1.01 \\
\hline ZBI Role Strain & 165 & $8(4.6)$ & $0-12$ & $0-12$ & 13.9 & 1.2 & 4.4 & $(2.9)$ & 0.22 \\
\hline \multicolumn{10}{|c|}{ No cognitive impairment ${ }^{b}$} \\
\hline DEMQOL Proxy & 57 & $0(0.0)$ & $31-124$ & $49-123$ & 0.0 & 0.0 & 89.9 & $(14.6)$ & -0.38 \\
\hline EQ-5D Proxy & 56 & $1(1.8)$ & $-0.594-1$ & $-0.003-1$ & 0.0 & 12.3 & 0.632 & $(0.262)$ & -0.71 \\
\hline EQ-5D Self & 53 & $4(7.0)$ & $-0.594-1$ & $-0.016-1$ & 0.0 & 47.4 & 0.842 & $(0.235)$ & -2.29 \\
\hline ZBI Total score & 52 & $5(8.8)$ & $0-48$ & $0-38$ & 5.3 & 0.0 & 12.5 & $(9.2)$ & 0.99 \\
\hline ZBI Personal Strain & 52 & $5(8.8)$ & $0-36$ & $0-28$ & 10.5 & 0.0 & 8.2 & $(7.1)$ & 1.24 \\
\hline ZBI Role Strain & 57 & $0(0.0)$ & $0-12$ & $0-10$ & 17.5 & 0.0 & 4.3 & $(3.0)$ & 0.11 \\
\hline \multicolumn{10}{|l|}{ Cognitive impairment ${ }^{\mathrm{c}}$} \\
\hline DEMQOL Proxy & 92 & $5(5.2)$ & $31-124$ & $38-111$ & 0.0 & 0.0 & 84.0 & $(16.6)$ & -0.50 \\
\hline EQ-5D Proxy & 89 & $8(8.2)$ & $-0.594-1$ & $-0.429-1$ & 0.0 & 6.2 & 0.554 & $(0.317)$ & -0.96 \\
\hline EQ-5D Self & 92 & $5(5.2)$ & $-0.594-1$ & $-0.594-1$ & 2.6 & 39.2 & 0.778 & $(0.306)$ & -2.24 \\
\hline ZBI Total score & 88 & $9(9.3)$ & $0-48$ & $0-48$ & 5.2 & 1.0 & 13.9 & $(10.0)$ & 1.00 \\
\hline ZBI Personal Strain & 88 & $9(9.3)$ & $0-36$ & $0-36$ & 11.3 & 1.0 & 9.5 & $(8.2)$ & 0.97 \\
\hline ZBI Role Strain & 90 & $7(7.2)$ & $0-12$ & $0-12$ & 10.3 & 2.1 & 4.4 & $(2.8)$ & 0.41 \\
\hline
\end{tabular}

Note. EQ-5D: index value based on UK TTO value set (high value = good health state). ZBI-Short version Personal Strain: items 1 - 9. ZBI-Short version Role strain: items 10 - 12 .

${ }^{\text {a }} N=173$.

${ }^{\mathrm{b}} n=57$.

${ }^{c} n=97$. 
Table 3

Convergent and Discriminant Validity of DEMQOL-Proxy (Self-Administered)

DEMQOL Proxy total score

r $(95 \% \mathrm{CI})$

\section{Convergent Validity:}

DEMQOL total score

$0.32 \quad(0.16 ; 0.47)$

EQ-5D Proxy-rating ${ }^{\mathrm{a}}$

$0.52 \quad(0.38 ; 0.65)$

Zarit Burden Interview

$\begin{array}{lll}\text { Total score } & -0.46 & (-0.61 ;-0.30) \\ \text { Personal Strain } & -0.41 & (-0.57 ;-0.24) \\ \text { Role Strain } & -0.45 & (-0.58 ;-0.28)\end{array}$

\section{Discriminant Validity:}

EQ-5D Self-rating ${ }^{a}$

Carer background variables

Gender

Age

Years acquainted

Hours spent (last week)

Patient background variables

Gender

Age
$0.20 \quad(0.02 ; 0.40)$

$-0.03 \quad(-0.19 ; 0.13)$

$0.20 \quad(0.05 ; 0.34)$

$0.06 \quad(-0.11 ; 0.23)$

$0.13 \quad(-0.05 ; 0.30)$

$-0.05 \quad(-0.22 ; 0.12)$

$0.08 \quad(-0.11 ; 0.27)$

Note. $N=139$. Pearson correlations and $95 \%$ CIs based on Simple Sample Method bootstrap analysis (case resampling with replacement from the original dataset, $N=1000$ ).

andex value based on UK TTO value set (high value $=$ good health state). 